\title{
Didáctica de la Competencia Gramatical mediada por Aprendizaje Activo en Estudiantes de una Licenciatura
}

\author{
Luz N. Molano(1)*, Ruby E. Mendoza(2) y Hugo H. Mendoza(3) \\ (1) Universidad Pedagógica y Tecnológica de Colombia. Facultad. Estudios a Distancia, Esc. Ciencias Humanísticas y \\ Educación, Av. Central del Norte, Tunja, Colombia (e-mail: luz.molano@uptc.edu.co) \\ (2) Universidad Pedagógica y Tecnológica de Colombia. Facultad. Estudios a Distancia, Esc. Ciencias Humanísticas y \\ Educación, Colombia. (e-mail: ruby.mendoza@uptc.edu.co) \\ (3) Universidad Pedagógica y Tecnológica de Colombia. Facultad. Estudios a Distancia, Esc. de Posgrados, Colombia. \\ (e-mail: hugo.mendoza@uptc.edu.co)
}

Recibido Ene. 30, 2019; Aceptado Mar. 19, 2019; Versión final Jun. 27, 2019, Publicado Dic. 2019

\begin{abstract}
Resumen
Se determina y analiza la incidencia de la intervención pedagógica Aprendizaje Basado en Problemas sustentada en principios de Aprendizaje Activo en el desempeño de la didáctica de la competencia gramatical en estudiantes del noveno semestre de una Licenciatura. El método de investigación empleado es mixto. Se aplicaron técnicas e instrumentos de corte cuantitativo para explicar la relación entre las variables Aprendizaje Activo y la competencia específica a través de la aplicación de una prueba y su análisis estadístico t-student. Es cualitativo porque describe la realidad inscrita en el aula desde sus participantes a través de la aplicación de instrumentos como cuestionarios, guías de entrevistas y diarios de campo. El diseño es experimental, tomando un grupo de control, aplicando una prueba pre y post. Después de la aplicación de la estrategia, se demostró que los estudiantes desarrollaron habilidades y atributos de aprendizaje activo. Se concluye que el aprendizaje activo como metodología y estrategia, permite el diseño, aplicación y evaluación de los procesos de enseñanza y aprendizaje.
\end{abstract}

Palabras clave: aprendizaje activo; aprendizaje basado en problemas; competencias en educación; estrategias educativas

\section{Development of Professional Skills through Problem-Based Learning}

\begin{abstract}
The incidence of the pedagogical intervention about Problem Based Learning based on Active Learning principles in the performance of the grammatical competence didactics in students of the ninth semester of a Bachelor's Degree is determined and analyzed. The research method used was mixed. Quantitative techniques and instruments were applied to explain the relationship between the Active Learning variables and the specific competence through the application and analysis of a t-student test. Is qualitative because it describes and understands the reality inscribed in the classroom from its participants through the application of instruments such as questionnaires, interview guides and field journals. The design is experimental, including a control group and a pre and post test was applied. After the implementation of the strategy, it was demonstrated that the students developed active learning skills and attributes. It is concluded that active learning as a methodology and strategy allows the design, application and evaluation of teaching and learning processes.
\end{abstract}

Keywords: active learning; problem-based learning; competences in education; educational strategies 


\section{INTRODUCCIÓN}

La intervención pedagógica Aprendizaje Basado en Problemas (ABP), se aplicó en la población objeto de estudio teniendo en cuenta los principios de Aprendizaje Activo propuestos por autores como Revans (1998); Koo (1999); Huber (2008) y Dewey (1998). Incluye la competencia específica de diseño y operacionalización de estrategias de enseñanza y aprendizaje, vinculada con la temática "Didáctica de la competencia gramatical". Es así como el Aprendizaje Activo (de aquí en adelante AA), como estrategia vinculada al modelo constructivista, requiere involucrar al estudiante en su proceso de aprendizaje y la planeación por parte del docente de situaciones didácticas que promuevan operaciones de orden superior como interpretación, análisis, síntesis, inferencia, evaluación, entre otras. Para Revans (1998) el aprendizaje activo "es una estrategia que se orienta a facilitar al diseño, implementación y evaluación de los procesos de enseñanza aprendizaje", de tal forma que a través de la interacción constante se construya el aprendizaje de manera cooperativa dando lugar para que a partir de la exploración de saberes previos, se generen experiencias significativas de aprendizaje.

Huber (2008), propone la aplicación del Aprendizaje Activo para la formación de ciudadanos dinámicos dentro de una sociedad democrática, para dar respuesta a los retos del mundo moderno dentro de un campo laboral que exige la apropiación de competencias para trabajar colaborativamente en busca de un bien común. Así, que este aprendizaje se convierte en un camino para lograr la formación de profesionales idóneos en cada campo del saber en el cual se están formando. Así mismo, el AA conlleva a la puesta en práctica de una serie de métodos que rompen totalmente con la rutina y enfoques reduccionistas en el aula, tales como el Aprendizaje Basado en Problemas (ABP), estudio de caso, trabajo por proyectos, entre otros; Peñalosa y Castañeda (2008, p. 8) al respecto mencionan "en un proceso activo, constructivo, a través del cual los estudiantes plantean metas de aprendizaje y después intentan monitorear, regular y controlar su cognición, su motivación y su conducta". Por lo que, el papel del discente es netamente activo y proactivo siendo la escuela el sitio idóneo para su desenvolvimiento personal y desarrollo integral, no solo para adquirir conocimientos.

Teóricos del AA como Schwartz y Pollishuke, (1995) y Koo (1999), plantean una nueva organización de la clase, no sólo como espacio físico, sino como un ambiente de aprendizaje donde se privilegien e integren nuevas alternativas de aprendizaje activo, desde la misma explicación docente hasta técnicas más avanzadas que requieren una metodología más cuidadosa y secuencial para dar respuesta a las exigencias del mundo moderno. La clase así vista debe contribuir al fortalecimiento de competencias específicas y genéricas como análisis, síntesis, aplicación de conocimientos en la práctica, conocimientos generales en el campo específico de estudio y habilidades interpersonales, para el trabajo autónomo y colaborativo, como lo plantea el Informe Tuning (2006).

Para garantizar que una intervención pedagógica cumpla con los principios del Aprendizaje Activo, es necesario tomar en cuenta los siguientes requisitos de Kolb (1976): experiencia concreta, obser-vación reflexiva; tiempo para reflexionar, observar, pensar sobre la experiencia que ha pasado y conceptualización abstracta. Así mismo, Involucra la formación de principios guía derivados de la reflexión sobre la experiencia y experimentación activa, consiste en proveer una oportunidad para tener la misma experiencia otra vez, pero con más entendimiento. Como toda metodología, el Aprendizaje Activo exige para su puesta en práctica, una serie de etapas que son comunes a casi todas las intervenciones didácticas aplicadas en el aula. Inicialmente, será necesario detectar los saberes previos de los estudiantes para determinar las necesidades de aprendizaje, después se entrará a reflexionar la experiencia personal para incrementar el entendimiento de la situación. Una vez analizada la situación, se procede a la aplicación de la intervención a través de la colaboración mutua y, finalmente se realizará una evaluación de los aprendizajes para identificar los elementos que se deben reorientar o en su defecto, buscar otros apoyos (Chirino, 2016).

La intervención pedagógica del ABP, está basada en principios de Aprendizaje Activo y Aprendizaje Colaborativo puesto que, a partir de la presentación de un problema, los estudiantes buscan colaborativamente a través de la consulta en diversas fuentes las soluciones al mismo, a la vez que, ponen en práctica una serie de habilidades comunicativas y competencias básicas y genéricas. Según Barrows (1986), el ABP es un método de aprendizaje basado en el principio de usar problemas como punto de partida para la adquisición e integración de nuevos conocimientos. Este sistema didáctico requiere que los estudiantes se involucren de forma activa en su propio aprendizaje hasta el punto de definir un escenario de formación autodirigida, pero con la ayuda del maestro, tal como se muestra en la figura 1.

El (ABP) como metodología activa, es el camino para desarrollar en los estudiantes de la licenciatura la competencia resolución de problemas a través de la solución de dificultades ligados a su praxis pedagógica de manera que se conviertan en protagonistas de su proceso de autoaprendizaje, por cuanto hace reflexionar al estudiante y lo motiva a ser él mismo con la ayuda de sus compañeros; esto es, un aprendizaje colaborativo, sin que el docente pierda su liderazgo (Serna y Díaz, 2013). 


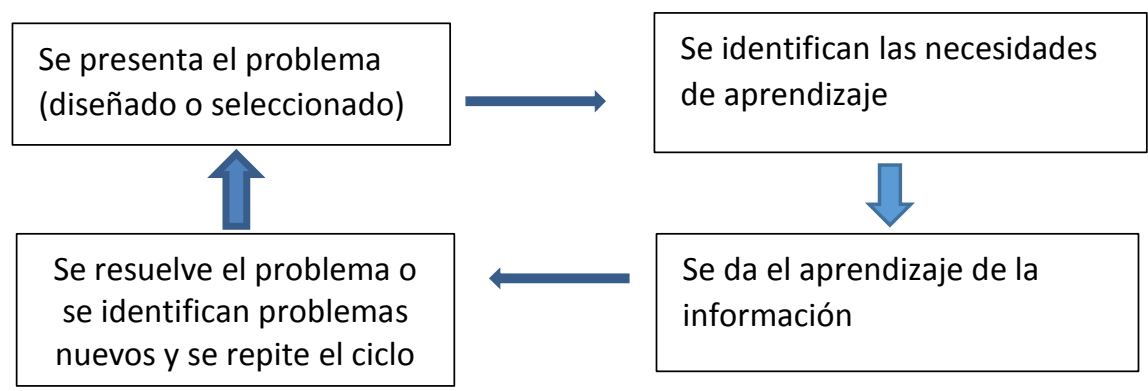

Fig. 1: Proceso de ABP. Adaptado de Instituto Tecnológico de Monterrey (2015)

Según Barrows y Tamblyn (2006), el ABP es un enfoque centrado en el estudiante con las siguientes características: el estudiante es quien decide qué necesita para aprender y quien identifica el problema y sus soluciones; el aprendizaje se produce en pequeños grupos, en forma colaborativa buscan en diferentes fuentes y de diversas formas, la solución al mismo. El docente presenta el problema con cierto nivel de dificultad y guía a los estudiantes para que desarrollen competencias críticas y reflexivas en torno a un trabajo común. El problema debe ser auténtico así como las soluciones. Para Walker, Andrew, Leary, Heather, Hmelo-Silver, Ertmer (2015), un aspecto importante relacionado con ABP es que este necesita integrar, en su ejecución una amplia gama de disciplinas por cuanto:

"Barrows notes that during self-directed learning, students should be able to access, study and integrate information from all the disciplines that might be related to understanding and resolving a particular problemjust as people in the real world must recall and apply information integrated from diverse sources in their work" (p. 8).

"Barrows (2008) señala que durante el aprendizaje autodirigido, los estudiantes deben estar en capacidad de acceder, estudiar e integrar información de todas las disciplinas que puedan estar relacionadas con la comprensión y la resolución de un problema particular, al igual que las personas en el mundo real deben recordar y aplicar información integrada desde diversos recursos en sus trabajos" Walker, Leary, Heather, Hmelo-Silver, Ertmer, $(2015$, p. 8) Traducción autores

Lo anterior implica que los estudiantes deben integrar en la solución del problema diferentes aristas, fuentes y perspectivas en la solución de problema que se plantea no solo en el área específica en la que se circunscribe, sino en diferentes disciplinas que puedan apoyarlo y que lo sustenten, así como la adaptación al contexto y necesidades que puedan surgir. Para el caso de la Educación Superior, y específicamente de los estudiantes de la licenciatura, el ABP permitió el desarrollo de pensamientos crítico y resolución de problemas, la inclusión de habilidades para el trabajo colaborativo, la búsqueda de información especializada y en fuentes confiables, así como el fortalecimiento de valores y actitudes para el trabajo en equipo, el aprendizaje autónomo y autodirigido.

Según el Espacio Europeo de Educación Superior (EEES), la educación superior debe propender por una formación basada en competencias genéricas y específicas. Las primeras son transferibles o transversales entre las diferentes profesiones y ocupaciones y las segundas son específicas a cada profesión o área del conocimiento. Para Villa y Poblete $(2007$, p. 23) "las competencias son factores de superación individual y grupal que permiten el desarrollo de los recursos personales para integrarlos en las posibilidades del entorno y obtener así, de esa complementariedad, el mayor beneficio mutuo". Para lo cual, establecen una taxonomía de competencias transversales clasificándolas en competencias genéricas instrumentales, interpersonales y sistémicas con sus correspondientes subcompetencias y que, según los autores, son una característica esencial y elemento clave de la Educación Superior. Por su parte, el Informe Tuning para América Latina, define las competencias en un ámbito genérico y citando a Delors éstas son "una combinación de atributos relacionados con la formación completa del conocimiento del sujeto, guiados por el desarrollo de habilidades prácticas, instrumentales y cognitivas fundamentales para satisfacer las exigencias de un mundo globalizado" (Ferreira, K. y Gomes Lima, 2013, p.6).

Para el caso de la investigación, se tomó el concepto de competencia dado por Cullen (1993) y consignado en el citado informe Tuning para América Latina, en el que se relaciona a las competencias con las capacidades integradas a la formación de los estudiantes con el fin de prepararlos para su desempeño social y laboral en un mundo globalizado que requiere profesionales con altas cualidades intelectuales, personales y sociales. El Proyecto Tuning, establece las competencias como la integración de conocimientos, habilidades y capacidades que adquiere el estudiante a lo largo de un programa, dentro de éstas, las específicas son aquellas relacionadas con las áreas del saber y que son cruciales para cualquier titulación; y referidas a la especificidad propia de cada campo, es decir, son la base del ejercicio profesional y están vinculadas a 
condiciones específicas de ejecución, por lo tanto no son tan fácilmente transferibles a otros contextos laborales, dadas sus particularidades. Para Yániz y Villardón (2006), estas competencias se derivan de la exigencia de un contexto o trabajo concreto y se forma en la interacción de la práctica docente con el contexto.

La competencia específica de diseño y operacionalización de estrategias de enseñanza y aprendizaje, consignada en el Proyecto Académico Educativo (PAE, 2009) de la Licenciatura en Educación Básica de la Universidad Pedagógica y Tecnológica de Colombia, busca que el futuro licenciado diseñe, aplique y evalúe estrategias de enseñanza con base en enfoques y metodologías contemporáneas encaminadas a formar estudiantes para la vida. Los estudiantes tendrán la oportunidad de trabajar colaborativamente en la resolución de problemas relacionados con la didáctica adecuada para trabajar la competencia gramatical en el nivel de básica secundaria.

Villa y Poblete (2007), establecen los siguientes niveles para el trabajo de esta competencia en contextos universitarios: el primero, la identificación y análisis del problema para generar alternativas de solución; en el segundo nivel se utiliza la experiencia y el criterio propio para analizar las causas y soluciones de un problema y, finalmente, proponer y construir en equipo soluciones a problemas en diversos ámbitos con una visión global. Para la presente investigación, se trabajó el nivel 3 en el cual se propone construir en equipo soluciones a un problema de la didáctica de la lengua Castellana con una visión global partiendo de un primer momento individual en el que los estudiantes analizan el problema para posteriormente construir las soluciones en forma colaborativa.

La competencia resolución de problemas, es indispensable para el desempeño laboral de los futuros licenciados por cuanto deben enfrentarse a situaciones complejas para las que se buscan alternativas de solución en las que ejerza su liderazgo en lo individual y grupal. Villa y Poblete (2007) indican que al demostrar tener unos criterios para abordar problemas y resolverlos, puede facilitar el desarrollo personal, la seguridad en sí mismo y el dominio del entorno.

En consecuencia, la evaluación de las competencias tanto específicas como genéricas debe ser continua y coherente con el proceso de aprendizaje y la metodología a seguir (clase expositiva, ABP, estudio de caso, trabajo por proyectos, seminarios, etc.) y debe hacer referencia a la aplicación del conocimiento en contextos reales (Riesco, 2008). Evaluar una competencia genérica implica hacer un juicio de valor sobre las prácticas que definen esta competencia, basándose en criterios de ejecución a partir de la evidencia (Yániz y Villardón, 2012). Para tal fin, es necesario conocer con claridad qué se va a evaluar y los distintos niveles de adquisición de la competencia en cuestión. Tales niveles están relacionados con tres criterios: a) conocimiento de la competencia que se va a evaluar, b) su aplicación en situaciones diversas y, c) transferencia a contextos reales tanto personales como profesionales y laborales Villa y Villa (2007, citados por Villarroel y Bruna, 2014).

Así es como el presente artículo describe la experiencia realizada con el fin de integrar y trabajar la competencia resolución de problemas a través de las etapas del ABP propuestos por autores como Villa y Poblete (2007); Escribano y Del Valle (2010), Garrigós y Valero (2012), tomando en cuenta que el ABP tiene entre sus propósitos ser una estrategia que promueve pensamiento crítico, creativo y reflexivo; desarrolla una visión holística de la realidad, estimula estudio independiente y autodirigido, genera el hábito para el trabajo en equipo, integra la capacidad de aprender a prender y la habilidad para solucionar problemas específicos de su entorno y profesión (Gutiérrez, Puente, Martínez y Piña, 2012). A través de la intervención se dio solución al problema planteado con respecto a la formación de las competencias resolución de problemas y operacionalización de la didáctica de la competencia gramatical.

\section{METODOLOGÍA}

El método de investigación que se empleó es de tipo mixto por cuanto se aplicaron técnicas e instrumentos de corte cuantitativo y cualitativo (Valenzuela y Flores, 2013) y, cuantitativo en la medida que explica de manera objetiva la relación entre las variables Aprendizaje Activo, y la competencia específica a través de la aplicación y análisis de una prueba $t$-student. Así mismo, de acuerdo con McMillan y Schumacher (2005), el diseño es de corte experimental pues se tomó un grupo de control y se le aplicó una prueba escrita pre y post, mediada por la intervención pedagógica, con el fin de comprobar si hubo o no cambios significativos en el grupo seleccionado. En relación con su naturaleza cualitativa la investigación describe y comprende la realidad inscrita en el aula desde sus participantes a través de la aplicación de instrumentos como cuestionarios, guías de entrevistas, rúbricas de evaluación, autoevaluación y coevaluación, así como diarios de campo producto de la observación sistémica y naturalista.

Con base en la investigación cualitativa, los participantes fueron seleccionados teniendo en cuenta las necesidades y el propósito del estudio que era determinar la incidencia del ABP sustentado en principios de Aprendizaje Activo para el desarrollo de competencias en estudiantes de IX semestre de una licenciatura. Los 
participantes son estudiantes de la Licenciatura en Educación Básica de la Facultad de Estudios a Distancia de la UPTC, pertenecen al Centro Regional de Atención a Distancia (CREAD) de la ciudad de Bogotá, IX semestre y cursan la asignatura Profundización en el área de Lengua Castellana.

\section{Instrumentos de recolección de la información:}

Para la recolección de la información y el análisis de los mismos se utilizaron instrumentos de medición tanto cuantitativos como cualitativos como guías de observación, diarios de campo, cuestionarios y guías de entrevista de tal manera que se dio respuesta a la pregunta y los objetivos de la investigación. Los instrumentos miden desempeños y actitudes individuales frente a la metodología de Aprendizaje Activo, a la intervención pedagógica Aprendizaje Basado en Problemas (ABP) y al trabajo con respecto a la formación de la Competencia resolución de problemas, así:

Examen de conocimientos: Este examen tipo opción múltiple con única respuesta fue elaborado por los investigadores, quienes son docentes de la Licenciatura, con el fin de explorar de saberes previos con respecto a la competencia específica operacionalización de estrategias de enseñanza-aprendizaje para la reflexión metalingüística y didáctica de la competencia gramatical en la asignatura Lengua Castellana. EI cuestionario previo constaba de 10 preguntas en las cuales se indagaba por conceptos básicos y clave en la didáctica de la gramática en el aula. Se aplicó también al final de la intervención, pero con preguntas más puntuales y aun nivel más profundo y, posteriormente se analizaron los resultados pre y post a través de una T-student

Test de competencia resolución de problemas: Para la evaluación y análisis de la competencia resolución de problemas, se tuvieron en cuenta los niveles, indicadores y descriptores de dominio propuestos por Villa y Poblete (2007, pp. 80-83), para esta competencia. Los autores proponen tres niveles de dominio con sus correspondientes indicadores y descriptores que van desde el 1 a 5 , siendo uno (1) el desempeño más bajo y cinco (5) el más alto. Se aplicó uno de entrada y otro al final, en este caso se tuvo en cuenta el nivel de dominio 3, por su estrecha relación con el ABP. El instrumento se aplicó al inicio y al final de la intervención.

Entrevista semiestructurada para aprendizaje activo en el aula: Este instrumento diseñado por Chirino (2017), está compuesto de preguntas abiertas y cerradas que buscaban determinar en primera instancia, un diagnóstico acerca de las percepciones de los estudiantes frente a las prácticas docentes y a los estilos de aprendizaje, ya sea situado, autorregulado o constructivo. Igualmente se aplicó al final de la intervención el instrumento de cierre el cual determinó de forma definitiva la incidencia del aprendizaje activo en el aprendizaje de los estudiantes frente a la competencia específica.

Diario de campo guía de observación del trabajo colaborativo. A través de este instrumento (Jaimes, 2014), se recogieron las percepciones y las observaciones realizadas por la investigadora a los largo de la aplicación de la intervención durante los espacios de trabajo colaborativo con respecto a eventos, situaciones y comportamientos de los estudiantes durante la resolución del problema en ABP.

Rúbricas de evaluación, coevaluación y autoevaluación de la intervención $A B P$ : A través de estos instrumentos de evaluación se midió y determinó cómo los estudiantes llevaron a cabo cada una de las fases de la intervención pedagógica Aprendizaje Basado en Problemas. Se aplicó un instrumento para autoevaluar el $A B P$, otro para evaluar el trabajo colaborativo teniendo en cuenta que esta es una metodología en la cual se trabaja la mayor parte del tiempo en equipo.

\section{RESULTADOS}

A continuación se presentan los resultados de la investigación, producto del análisis de los instrumentos aplicados, antes, durante y después de la intervención pedagógica basada en principios de Aprendizaje Activo. Los instrumentos diseñados tienen relación con las variables Aprendizaje Activo, Aprendizaje Basado en Problemas, competencia específica operacionalización de la didáctica de la competencia gramatical y competencia genérica resolución de problemas, aplicados a los estudiantes objeto de estudio durante el trabajo de campo.

La competencia específica operacionalización de la didáctica de la competencia gramatical, se vincula con la asignatura Profundización en el área de Lengua Castellana, perteneciente al IX semestre de la Licenciatura en Educación Básica. Su importancia radica en que la gramática ha sido uno de los temas álgidos para abordar en el aula y los docentes en formación deben tener clara cuál es la didáctica adecuada para su trabajo con los estudiantes de tal manera que se fomente una gramática vinculada al texto (Ochoa, 2008)

En la tabla 1, se sintetiza la experiencia vivida en la intervención. 
Tabla 1. Descripción sintética del proceso de aprendizaje activo conforme a principios e investigación acción.

\begin{tabular}{|c|c|c|c|}
\hline $\begin{array}{l}\text { Fases de la } \\
\text { metodología de } \\
\text { aprendizaje activo }\end{array}$ & $\begin{array}{l}\text { Síntesis de tareas } \\
\text { planeadas }\end{array}$ & $\begin{array}{l}\text { Modificaciones } \\
\text { sustantivas en la acción }\end{array}$ & $\begin{array}{l}\text { Justificación de las modificaciones } \\
\text { ¿Cuál fue la causa que hizo } \\
\text { necesario modificar lo planeado }\end{array}$ \\
\hline Contextualización & $\begin{array}{l}\text { Aplicación de una Evaluación } \\
\text { diagnóstica del tema y pre test } \\
\text { con escala tipo Likert de la } \\
\text { competencia pensamiento } \\
\text { resolución de problemas. } \\
\text { Explicación magistral con una } \\
\text { presentación en power point } \\
\text { Aplicación del Instrumento } \\
\text { diagnóstico Aprendizaje Activo } \\
\text { en el aula. } \\
\text { Creación de Foro de discusión }\end{array}$ & $\begin{array}{l}\text { Ajustes a la evaluación } \\
\text { diagnóstica. } \\
\text { Se creó el foro, pero la } \\
\text { participación se enfocó } \\
\text { más hacia preguntas o } \\
\text { dudas. }\end{array}$ & $\begin{array}{l}\text { Se ajustó con el fin de que esta } \\
\text { realmente evaluara competencia } \\
\text { específica, no sólo saberes. } \\
\text { Los estudiantes tienden a generar } \\
\text { peguntas más que respuestas } \\
\text { inicialmente. }\end{array}$ \\
\hline Reflexión individual & $\begin{array}{l}\text { Exploración de saberes } \\
\text { previos. Dar respuesta a los } \\
\text { interrogantes, planteados por } \\
\text { Huber (2008). } \\
\text { Realimentación del tema }\end{array}$ & $\begin{array}{l}\text { Las preguntas de } \\
\text { reflexión individual } \\
\text { fueron dejadas en el foro } \\
\text { virtual. }\end{array}$ & $\begin{array}{l}\text { Los encuentros con los estudiantes } \\
\text { son cada quince días, fue necesario } \\
\text { emplear la plataforma virtual. }\end{array}$ \\
\hline Acción - Práctica & $\begin{array}{l}\text { Selección de una situación } \\
\text { problema relacionada con la } \\
\text { didáctica de la competencia } \\
\text { gramatical. } \\
\text { Monitorear y orientar las } \\
\text { reglas de la actividad y el } \\
\text { trabajo en equipo de forma } \\
\text { presencial y virtual a través } \\
\text { del foro. } \\
\text { Establecer tiempos para que } \\
\text { los estudiantes resuelvan el } \\
\text { problema. } \\
\text { Organizar sesiones de tutoría } \\
\text { individual y/o grupal. } \\
\text { Asignación de roles: } \\
\text { moderador, relator, } \\
\text { participantes. } \\
\text { Diseño de matriz de } \\
\text { seguimiento de acuerdo con } \\
\text { las fases del ABP, }\end{array}$ & $\begin{array}{l}\text { El trabajo colaborativo } \\
\text { para la resolución del } \\
\text { problema en ABP, se } \\
\text { realizó principalmente a } \\
\text { través de Google Drive. }\end{array}$ & $\begin{array}{l}\text { No fue posible la creación de } \\
\text { grupos de trabajo en la Plataforma } \\
\text { Moodle, debido a la existencia de } \\
\text { grupos por cada una de las sedes } \\
\text { pertenecientes al Programa. }\end{array}$ \\
\hline Reflexión Colectiva & $\begin{array}{l}\text { Se enfoca la reflexión a través } \\
\text { de preguntas. } \\
\text { Aplicación de Rúbrica de } \\
\text { coevaluación del trabajo } \\
\text { grupal y responsabilidad } \\
\text { individual. }\end{array}$ & No hubo modificaciones. & \\
\hline Evaluación & $\begin{array}{l}\text { Diseño de evaluación } \\
\text { sumativa del tema para } \\
\text { comprobar la formación de la } \\
\text { competencia } \\
\text { operacionalización de la } \\
\text { didáctica de la competencia } \\
\text { gramatical. } \\
\text { Evaluación de la competencia } \\
\text { genérica a través de rúbrica. } \\
\text { Evaluación de la metodología } \\
\text { de aprendizaje activo por } \\
\text { medio de entrevista. }\end{array}$ & No hubo modificaciones & \\
\hline Mejora continua & $\begin{array}{l}\text { Realimentación continua en el } \\
\text { Drive de cada grupo y del } \\
\text { informe final. }\end{array}$ & No hubo modificaciones & \\
\hline
\end{tabular}


La tabla 1 presenta una síntesis del trabajo de campo realizado de acuerdo con la propuesta para el diseño para intervenciones pedagógicas de Chirino, Ramos y Lozano. (2014). Esta parte muestra, en primera instancia, la síntesis de tareas planeadas para cada una de las fases de aprendizaje activo, a saber: la contextualización por parte del docente en la cual se exploran saberes previos y se introduce el tema que guiará la intervención, en este caso didáctica de la competencia gramatical. Posteriormente se realizó una reflexión individual con base en las preguntas de Huber (2008). Igualmente la tabla explica y justifica algunas modificaciones que tuvieron que realizarse a lo inicialmente planeado como el cambio de herramienta sincrónica foro para el trabajo colaborativo, en el Google Drive, debido a dificultades técnicas en el Aula Virtual de la UPTC para la conformación de los grupos.

La figura 2 presenta las fases del trabajo de campo realizado de acuerdo con la propuesta para intervenciones pedagógicas de Chirino, Ramos y Lozano. (2014). Esta parte muestra, en primera instancia, la síntesis de tareas planeadas para cada una de las fases de aprendizaje activo, a saber: la contextualización por parte del docente en la cual se exploran saberes previos y se introduce el tema que guiará la intervención, en este caso didáctica de la competencia gramatical. Posteriormente se realizó una reflexión individual con base en las preguntas de Huber (2008). Le sigue la explicación de la acción práctica o trabajo de campo, propiamente dicho que involucra la aplicación en el aula y a través del Google Drive de los pasos y fases de la estrategia ABP y técnicas seleccionadas, para posteriormente realizar una reflexión colectiva e individual por medio de instrumentos de evaluación y finalmente, de acuerdo con los resultados, continuar con las acciones de mejoramiento.

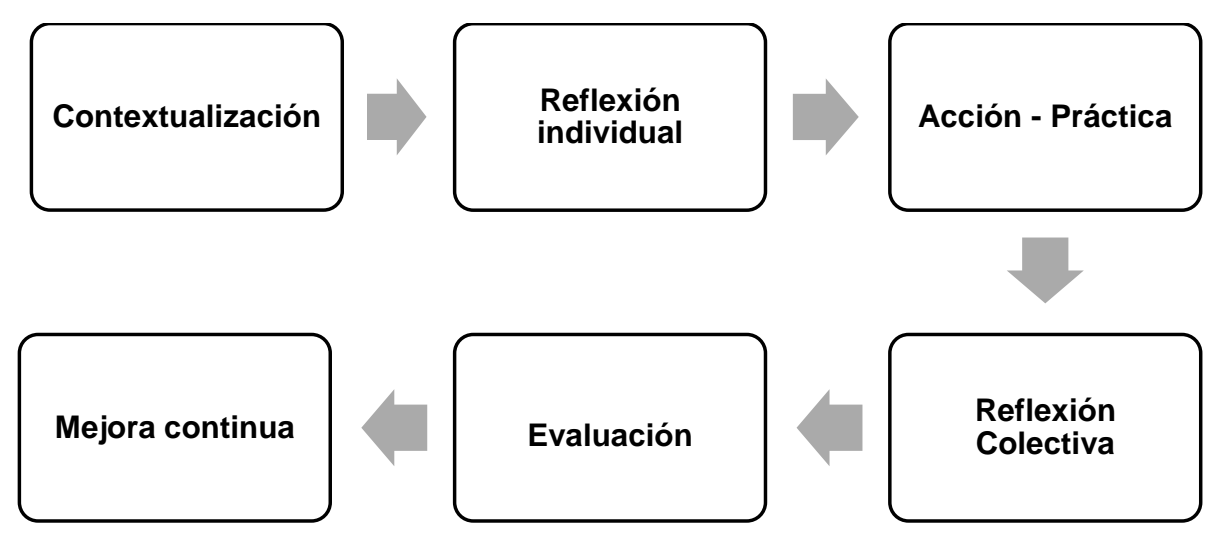

Fig. 2: Fases de la estrategia con base en Aprendizaje Activo. Adaptado de Chirino et al. (2014)

Con respecto al fortalecimiento de la competencia específica, los estudiantes mejoraron su desempeño en cuanto aplicaron a través de la metodología ABP, la didáctica adecuada para el trabajo de la competencia gramatical en el aula. El trabajo colaborativo a través del google drive permitió el compartir de saberes y experiencias para la resolución del problema relacionado con el tema. Los resultados de la prueba de saberes específicos arrojaron datos positivos en relación con los saberes previos y finales. Inicialmente se aplicó un cuestionario (diseñado por los investigadores), el cual determinó que los saberes previos de los estudiantes con respecto al tema, eran muy incipientes ya que los resultados fueron muy bajos como se mostrará más adelante en la figura 3. Posterior a la intervención, se volvió a aplicar el cuestionario y los resultados variaron positivamente con respecto a los conocimientos adquiridos en la didáctica de la competencia gramatical, aunque hubo casos en que la diferencia no fue significativa. Es importante aclarar que los cuestionarios se aplicaron con una diferencia de dos meses entre uno y otro, mediados por el trabajo colaborativo en el google drive. Al realizar el análisis de los resultados del cuestionario final, los estudiantes manifestaron que algunas preguntas habían estado lejos de su comprensión, pues no estaban en los documentos suministrados. Lo anterior porque algunas preguntas eran situaciones hipotéticas respecto a la didáctica de la gramática en situaciones específicas de aprendizaje, como por ejemplo qué debían hacer frente a dificultades con el uso del "de que" o qué materiales debían emplear para explicar la concordancia entre sujeto y predicado. En la tabla 2, se presenta el análisis cuantitativo aplicado a través del procedimiento de contraste entre el cuestionario inicial y final empleando para ello la prueba $T$-student.

La tabla 2 refleja los resultados estadísticos de tomar una muestra de 22 estudiantes con edades entre 21 y 38 años con un promedio de 28 años con desviación de 5 años. De los cuales el $28 \%$ son hombres y un $72 \%$ de mujeres, con los que se les aplicó una prueba de conocimientos en la competencia específica y se verifica que existe diferencia significativa estadística al aplicar el tratamiento descrito en el estudio. Antes de realizar la prueba de diferencias significativas, se debe comprobar que las variables presenten una distribución normal y varianza constante, para lo cual se aplicó el test de normalidad de Kolmogorov-Smirnov y el test de Levene para homogeneidad en la varianza. Los valores de probabilidad Sig. del estadístico de Kolmogorov - Smirnov 
0,56 y 0,082 mayores que 0,05 nivel de significancia al cual se compara, indican que no se rechaza la hipótesis de normalidad, significa que en las pruebas aplicadas a los estudiantes al inicio y final se distribuyen normal.

Tabla 2. Estadísticos Descriptivos

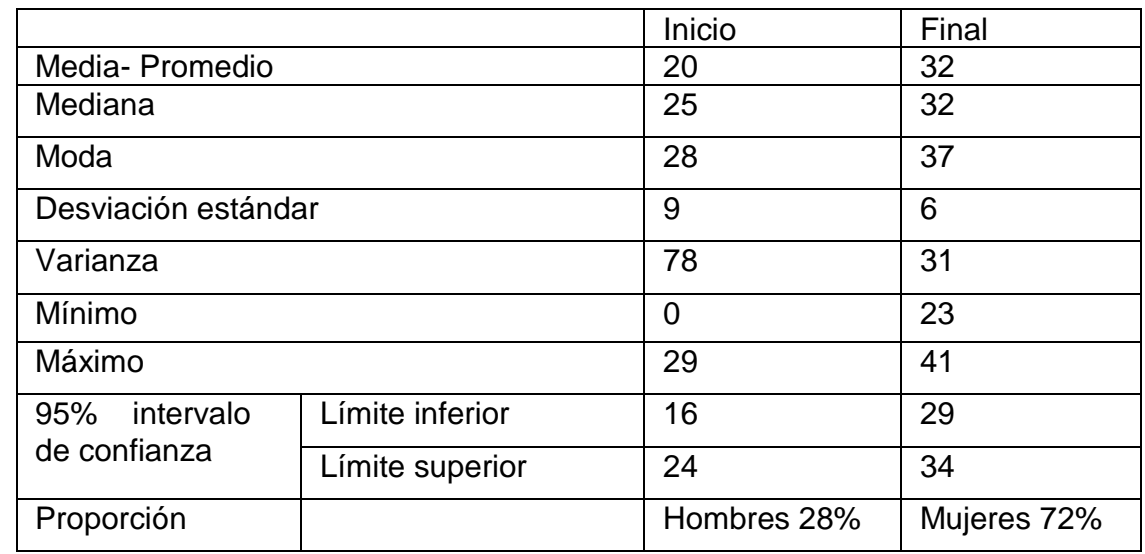

Los valores de probabilidad Sig. del estadístico de Levene 0,387 y 0,417 son mayores que 0,05 nivel de significancia al cual se compara, indican que no se rechaza la hipótesis de homoscedasticidad de la varianza en las variables puntaje obtenido al inicio y final. Cumplidas las dos condiciones anteriores de normalidad y homoscedasticidad se prueba la diferencia emparejada de promedios, mediante el estadístico $t$ de student para muestras relacionadas como se muestra en la tabla 3.

Tabla 3. Prueba diferencia de promedios en la competencia específica

\begin{tabular}{|c|c|c|c|c|c|c|c|}
\hline \multicolumn{5}{|c|}{ Diferencias emparejadas - Inicio - Final } & \multirow[t]{3}{*}{$\mathrm{t}$} & \multirow[t]{3}{*}{$\overline{g l}$} & \multirow{3}{*}{$\begin{array}{l}\text { Sig. } \\
\text { (bilateral) }\end{array}$} \\
\hline \multirow[t]{2}{*}{ Media } & \multirow[t]{2}{*}{$\begin{array}{l}\text { Desviación } \\
\text { estándar }\end{array}$} & \multirow{2}{*}{$\begin{array}{l}\text { Media de } \\
\text { error } \\
\text { estándar }\end{array}$} & \multicolumn{2}{|c|}{$\begin{array}{l}95 \% \text { de intervalo de } \\
\text { confianza de la diferencia }\end{array}$} & & & \\
\hline & & & Inferior & Superior & & & \\
\hline$-11,40909$ & 8,32471 & 1,77484 & $-15,10006$ & $-7,71812$ & $-6,428$ & 21 & ,000 \\
\hline
\end{tabular}

El valor de probabilidad del estadístico $t$ igual a cero (0) es menor que 0,05 nivel de significancia al cual se compara, indica que se rechaza la hipótesis nula de que los promedios al inicio de las pruebas y después de la intervención pedagógica sean iguales. Para aceptar la hipótesis alternativa o de estudio de que los promedios son diferentes. Lo anterior evidencia que la intervención aumenta significativamente el promedio, de 20 a 32 como se muestra en la figura 3.

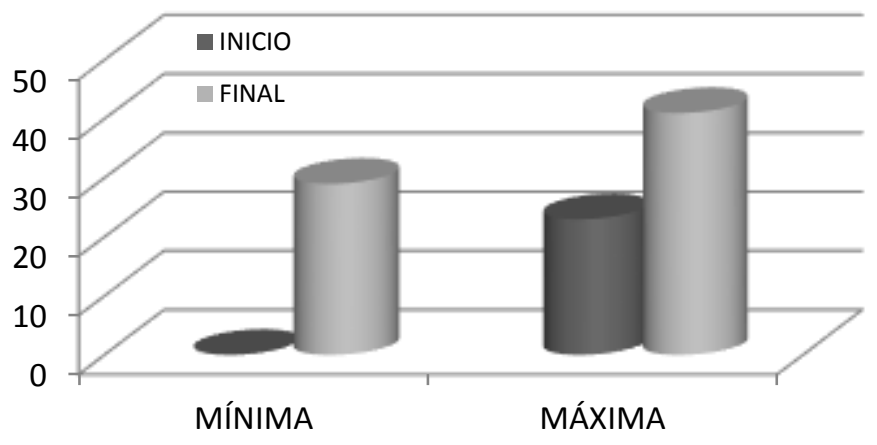

Fig. 3: Resultados prueba inicial

En la figura 3 se puede observar la diferencia en resultados respecto a la prueba inicial con calificaciones desde 0 hasta 2,3 producto de los escasos o nulos saberes previos de los estudiantes con respecto a la didáctica de la competencia gramatical, pese a que en semestres anteriores ya se había abordado el tema, por su estrecha relación con el proceso de producción textual. El resultado posterior a la intervención, aunque no fue el esperado, mejoró puesto que los resultados mínimos estuvieron por encima de 2,9 y la máxima fue de 4.1, con un promedio de 3.2. En esta oportunidad las preguntas fueron más acertadas con relación al tema, pero aún hay vacíos relacionados con situaciones específicas en el aula, que permiten inferir que falta mayor articulación entre la teoría y la práctica. 
Con los estudiantes que lograron los mayores porcentajes (coinciden en ser los mejores puntajes pre y pos), se realizó un contraste en relación con respuestas en la entrevista y estas fueron más contundentes respecto a la importancia del tema para su vida profesional y el beneficio para los estudiantes, pues consideran que la gramática "es un componente esencial de la lengua y su función es el uso correcto de la misma en situaciones de comunicación", así mismo, otra respuesta considera que el tema no solo aplica para el área de Lengua Castellana sino que "es transversal y se puede integrar en cualquier área del conocimiento" o "se parte de aprendizajes y experiencias significativas con otros compañeros de la institución donde trabajo" Al contrario, los estudiantes con los promedios de calificación muy bajos, dieron respuestas muy vagas e imprecisas a lo largo de toda la entrevista "no tengo claro el tema" , "creo que era sobre trabajo colaborativo" o "hay otras formas más interesantes de abordarlo" y no tienen clara la importancia del tema en su vida profesional.

Para la evaluación de la competencia genérica resolución de problemas se aplicó un cuestionario tipo Likert (con base en los niveles de Villa y Poblete, 2007), entre 0 y 5 para que el estudiante estableciera la frecuencia que consideraba aplicaba en su caso para los criterios relacionados con el desarrollo de la competencia genérica resolución de problemas en el nivel tres en donde 1 corresponde a nunca ocurre; 2 , raras veces; 3 , algunas veces; 4 , casi siempre y 5 , siempre. Una vez aplicados los cuestionarios previos y post se procedió a realizar la sistematización indicador por indicador, para posteriormente dar un resultado final que se consigna en la tabla 4.

Tabla 4. Comparativo de niveles de desempeño pre y post de la competencia transversal resolución de problemas Adaptado de Villa y Poblete (2017)

\begin{tabular}{|l|c|c|c|}
\hline & \multicolumn{2}{|l|}{ Promedio de Niveles de desempeño } \\
\hline Indicadores de desempeño & Previo & Posterior & Diferencia \\
\hline $\begin{array}{l}\text { Identifica los problemas con anticipación antes de que su } \\
\text { efecto se haga evidente. }\end{array}$ & 3 & 4 & 1 \\
\hline $\begin{array}{l}\text { Analiza los problemas y sus causas desde un enfoque } \\
\text { global y de mediano y largo plazo. }\end{array}$ & 3 & 4 & 0 \\
\hline $\begin{array}{l}\text { Dirige el proceso sistemático para la toma de decisiones } \\
\text { en grupo }\end{array}$ & 4 & 4 & 0 \\
\hline $\begin{array}{l}\text { Transfiere aprendizajes de casos y ejercicios de aula a } \\
\text { situaciones reales de otros ámbitos }\end{array}$ & 4 & 4 & 1 \\
\hline $\begin{array}{l}\text { Obtiene el respaldo de otros para apoyar sus acciones y } \\
\text { tener los suficientes aliados para el éxito de sus } \\
\text { decisiones }\end{array}$ & 3 & 4 & \multirow{2}{*}{} \\
\hline
\end{tabular}

En general se evidencia un fortalecimiento de la competencia genérica resolución de problemas después de la aplicación de la estrategia. Así mismo la guía de observación y la coevaluación del aprendizaje colaborativo en $A B P$, determinaron que los estudiantes trabajaron mancomunadamente en la resolución del problema, sortearon las dificultades y conflictos presentados en el proceso, existió un compromiso hacia un enfoque común y consideran que el rendimiento y grado de satisfacción del grupo fue adecuado. Así mismo algunos estudiantes manifiestan que no todos los integrantes tuvieron el mismo grado de responsabilidad ni la aceptación de las ideas de otros.

El aprendizaje activo como metodología y estrategia vinculada con el constructivismo, permitió el diseño, aplicación y evaluación de los procesos de enseñanza y aprendizaje de los estudiantes objeto de la investigación tal como lo platea Revans (1998). Además, es un aprendizaje que se visualiza a través de la experiencia y la construcción mediada por procesos de comunicación e interacción en la cual todos aprenden de manera significativa (Schwartz y Pollishuke, 1995), por medio del trabajo colaborativo dentro y fuera del aula tal como se aplicó a través de las fases del ABP, y que a su vez están directamente relacionadas con las fases de aprendizaje activo propuestas por Chirino, Ramos y Lozano (2014), con base en los estudios de Huber (2008).

\section{Impacto del proceso de Aprendizaje Activo en los alumnos}

A través del trabajo de campo, mediado por la tecnología (google drive) y las sesiones presenciales, los estudiantes desarrollaron habilidades para trabajar colaborativamente tal como lo plantean Escribano y Del Valle (2011): "en ABP, la estrategia está concebida para que el alumno desarrolle habilidades para analizar un problema en forma metódica, para desempeñar con éxito distintas funciones e incluso actividades individuales" (P. 22). Lo anterior permitió la construcción del conocimiento de forma significativa, a la vez que se propiciaron espacios para la reflexión tanto individual como grupal, a partir de las respuestas en el foro y en el instrumento previo (Chirino, 2017), para explorar saberes previos en los estudiantes y conocer sus expectativas frente al aprendizaje nuevo. 
Respuestas como: "El tema de la competencia gramatical es fundamental ya que nosotros como docentes debemos enseñar a los estudiantes todas las normas y orientarlos diariamente a que escriban bien y utilicen la palabras correctamente en su momento" o "Ser docente conlleva la responsabilidad de conocer y aplicar las reglas ortográficas en todas las áreas de conocimiento, por ende es importante aprenderlas y aplicarlas en todos los campos de la vida porque eres el ejemplo a seguir", demuestran que hubo un proceso de reflexión previo y posterior a la intervención pedagógica mediada por principios de aprendizaje activo. La tabla 5 muestra las variables relacionadas con el impacto del aprendizaje activo como estrategia de enseñanza aprendizaje.

Tabla 5. Definición de categorías relacionadas con el impacto del aprendizaje activo como estrategia de enseñanzaaprendizaje. (Adaptado, Chirino, 2017)

\begin{tabular}{|c|c|c|}
\hline Categoría & Verificación de aplicación en el aula & $\begin{array}{l}\text { Verificación de impacto en estrategia de } \\
\text { aprendizaje aplicada por los alumnos }\end{array}$ \\
\hline Activo & $\begin{array}{l}\text { Aplica metodología que integra etapas definidas } \\
\text { para estructurar una intervención didáctica, } \\
\text { incluye contextualización teórica, actividad, } \\
\text { reflexión individual, reflexión colectiva, vuelta a la } \\
\text { acción y evaluación diagnóstica y sumativa. } \\
\text { Puede incluir uno o varios ciclos }\end{array}$ & \\
\hline Autorregulado & & $\begin{array}{l}\text { Los estudiantes comprenden el sentido de lo que } \\
\text { hacen en el aula, conocen e incluso definen sus } \\
\text { metas de aprendizaje, son capaces de identificar } \\
\text { lo que no han logrado comprender o lo que no } \\
\text { saben hacer y pueden buscar cubrir sus } \\
\text { deficiencias por distintos medios. Buscan } \\
\text { nuevas fuentes de conocimiento (Huber, 1997). }\end{array}$ \\
\hline Constructivo & & $\begin{array}{l}\text { El conocimiento individual, al menos en parte, es } \\
\text { una construcción personal. Los estudiantes } \\
\text { construyen su conocimiento sobre todo } \\
\text { interpretando sus percepciones o experiencias, } \\
\text { dependiendo de sus conocimientos u opiniones } \\
\text { disponibles (Huber, 2008, p.66) }\end{array}$ \\
\hline Situado & $\begin{array}{l}\text { El contexto es aplicado al inicio de la intervención } \\
\text { didáctica y se dirige a reforzar los saberes previos } \\
\text { requeridos, a enfocar la comprensión de los } \\
\text { conocimientos básicos para realizar la actividad y } \\
\text { a dejar claras las oportunidades de aplicar los } \\
\text { conocimientos adquiridos en la realidad. } \\
\text { (Jonassen, Mayes \& McAleese, 1993, en Huber, } \\
2008, \text { p 66). }\end{array}$ & $\begin{array}{l}\text { El alumno comprende la importancia del } \\
\text { conocimiento adquirido en función de su } \\
\text { aplicabilidad en contextos reales. }\end{array}$ \\
\hline Social & $\begin{array}{l}\text { El aprendizaje se apoya en interacción con pares } \\
\text { y con docente. Cada proceso de enseñanza y } \\
\text { aprendizaje es una interacción social. Rogoff } \\
\text { (1995) I plano social, el plano interpersonal y el } \\
\text { plano personal. } \\
\text { Situación social = se presenta un modelo de } \\
\text { actividades a realizar que vincule de forma } \\
\text { participativa a alumnos con distintas destreza } \\
\text { Existe una guía, objetiva (en documento de algún } \\
\text { tipo) en donde se definan etapas y sistemas de } \\
\text { definidos a priori por el docente para la } \\
\text { participación de los alumnos su coordinación de } \\
\text { esfuerzos y formas de motivación alineados a los } \\
\text { valores del grupo y la institución. }\end{array}$ & $\begin{array}{l}\text { Existe disposición para trabajar con sus pares, lo } \\
\text { llegan a hacer en forma intencional, aunque no } \\
\text { se les pida. } \\
\text { La apropiación por participación se da en el al } \\
\text { plano personal, se identifica a partir de observar } \\
\text { la forma en la que los alumnos se transforman } \\
\text { en el desarrollo de la intervención didáctica. } \\
\text { Rogoff (1995) declara que eso es más un } \\
\text { proceso de desarrollo que un proceso de } \\
\text { adquisición.p.70 }\end{array}$ \\
\hline Reflexivo & $\begin{array}{l}\text { Según Jay y Johnson (2002) los estudiantes } \\
\text { deberían aprender no solamente como se } \\
\text { reflexiona sobre el objeto de aprendizaje y los } \\
\text { propios procesos de aprenderlo, sino también } \\
\text { sobre perspectivas o marcos alternativos y las } \\
\text { implicaciones de lo que se aprende. Los docentes } \\
\text { integran preguntas que orienten la reflexión al } \\
\text { menos una vez para el plano individual y para el } \\
\text { colectivo. (Huber, 2008, p. } 72 \text { ) }\end{array}$ & $\begin{array}{l}\text { En la reflexión los alumnos identifican tanto } \\
\text { hechos centrales como preguntas abiertas } \\
\text { respecto al objeto de aprendizaje. Lo mismo vale } \\
\text { en cuanto a las propias ideas, emociones, } \\
\text { resistencias, valores y preferencias. } \\
\text { La reflexión común ayuda a que se puedan } \\
\text { enterar de perspectivas alternativas }\end{array}$ \\
\hline
\end{tabular}


Previo a la intervención pedagógica se aplicó un cuestionario cualitativo de Aprendizaje Activo en el aula Chirino (2017) el cual indagaba a los estudiantes por la frecuencia de algunas acciones del docente y que su vez daban cuenta de la forma cómo los estudiantes aprenden dentro y fuera del aula. El análisis determinó que el $52 \%$ de los estudiantes presentan aprendizaje autorregulado ya que buscan información adicional a la proporcionada por el docente, mientras que el $48 \%$ se limitan a utilizar la información y explicaciones brindadas por el docente en el aula y en la Plataforma Virtual; un $74 \%$ de los estudiantes demuestran aprendizaje constructivo toda vez que piden explicaciones adicionales y buscan otras formas de comunicación entre sus pares, a diferencia del $26 \%$ que lo hace con menos frecuencia o sólo en algunas asignaturas. Con respecto al aprendizaje autorregulado el $76 \%$ de los encuestados manifestó que ellos mismos abren grupos de estudio en la mayoría de las asignaturas para trabajar colaborativamente, sin ser asignados por el docente.

Posterior a la intervención se aplicó un cuestionario cualitativo de salida Chirino (2017) el cual indagaba por los estilos de aprendizaje, según sea constructivo, situado o autorregulado en relación con la intervención pedagógica aplicada. El $77 \%$ de los estudiantes encuestados, coincide con lo esperado respecto a que tienen claro dónde van a aplicar lo aprendido una vez terminen sus estudios. Algunas respuestas significativas son que lo aplicarán en sus sitios de trabajo ya que la mayoría son docentes en ejercicio, consideran que lo aprendido desarrolla en ellos competencias laborales y disciplinares que posteriormente llevarán al aula con sus estudiantes. Igualmente consideran que no solo aprendieron a elaborar una secuencia didáctica para la formación de la didáctica de la competencia gramatical, sino que también aprendieron a trabajar en grupo y a liderar una actividad en la solución de un problema. Este tipo de respuestas son evidencia de que se logró un aprendizaje significativo puesto que, a una mayoría, al 73\% le quedó claro la importancia del tema en su desempeño docente para lograr la formación de la competencia gramatical en sus estudiantes.

Al contrario, algunos estudiantes que representan el $33 \%$ de la población, a pesar que consideran que el tema es importante, no se centraron en el desarrollo de la competencia específica sino en el trabajo colaborativo con la técnica $A B P$, es decir que para ellos fue más significativo la forma de trabajar que el tema central de la intervención. Existe una estrecha relación entre los estudiantes que obtuvieron los menores porcentajes en la prueba con las respuestas sistematizadas en el instrumento de Aprendizaje Activo tabla de vaciado. Son estudiantes que se conforman con las explicaciones del docente, no buscan otras fuentes de información o inclusive no tenían claro el tema durante ni después de la intervención y se les dificultó trabajar colaborativamente.

El $\mathrm{ABP}$ es un método didáctico, que cae en el dominio de las pedagogías activas y más particularmente en la estrategia de la enseñanza denominada aprendizaje por descubrimiento y construcción, que se contrapone a la estrategia expositiva o magistral, es un método basado en el principio de usar problemas como punto de partida para la adquisición e integración de los nuevos conocimientos. Barrows (2008), indica que el propósito de este principio es que el estudiante sea actor principal y responsable de su propio aprendizaje. Por consiguiente, al estar el proceso centrado en el estudiante hace que el docente adquiera el papel de facilitador; de igual forma, destaca que el núcleo de generación organizacional y de aprendizaje radica en la proposición de problemas, por cuanto estos desarrollan habilidades de pensamiento de nivel superior.

En este aspecto, una vez aplicado el cuestionario posterior a la intervención (Chirino, 2017), el 76\% de los estudiantes manifestaron que realizan actividades de autorregulación y aprendizaje constructivo como buscar información adicional a la proporcionada por el docente, pues consideran que es necesaria para lograr un mejor entendimiento del tema y para la solución del problema planteado; así mismo el $77 \%$ de los estudiantes vieron la importancia de abrir su propio grupo de estudio, independiente del proporcionado por la investigadora en el Aula y google drive, para ,mantenerse comunicados y mantener un trabajo colaborativo permanente ya que todos tienen whatsapp en sus celulares. Al contrario, un $33 \%$ de los encuestados piensa que no es indispensable buscar información adicional ni participaron de otros grupos abiertos en las redes sociales.

Para Huber (2008) tanto el enfoque situado como social conllevan a aprendizajes que parten de un momento individual y luego colaborativo mediado por el docente en los cuales se ponen en juego sus saberes previos. En el enfoque social influyen muchos procesos socioculturales en los cuales es importante el contexto donde se pondrán en práctica los conocimientos adquiridos. En el instrumento de AA, posterior a la intervención, los estudiantes en un $77 \%$ coinciden en reconocer que la docente realizó una exploraron de saberes previos y un $86 \%$ que realizó una contextualización del tema específico para determinar áreas de oportunidad y debilidad frente al tema y la competencia operacionalización de la didáctica de la competencia gramatical. En lo social, la metodología ABP parte de principios de aprendizaje colaborativo para la resolución del problema y permite según Serna y Diaz (2013) que los estudiantes tomen sus propias decisiones sin que el docente pierda su liderazgo en el aula, ya que éste debe estar al tanto de lo hacen sus estudiantes durante todo el proceso para guiarlos y reorientarlos cuando sea necesario y así lo reconoce el $100 \%$ de los estudiantes. 
Chirino, Ramos y Lozano (2014), indican que las actividades de reflexión buscan generar meta-aprendizajes relacionados con habilidades de aprendizaje autorregulado y aprender haciendo. La reflexión forma a su vez parte importante en la planeación didáctica de la estrategia basada en principios de aprendizaje activo (ver tabla 9). Tanto al inicio como al final se promueven espacios de reflexión individual y grupal en la que los estudiantes aplican metacognición y competencias de pensamiento crítico. Sin embargo en este aspecto solo 15 estudiantes, que corresponde al $68 \%$ creen que se propició la reflexión, mientras que el $32 \%$ manifiestan que faltaron espacios en el aula de clase para "aclarar dudas con la docente y platicar con los compañeros sobre las dificultades presentadas" o "es muy poco el tiempo que tuvimos para expresar cómo nos sentíamos ante una forma nueva de trabajar".

Triangulación: Partiendo de que la triangulación se refiere al uso de varios métodos (tanto cuantitativos como cualitativos), de fuentes de datos, de teorías, de investigadores o de ambientes en el estudio de un fenómeno (Okuda y Gómez, 2005), se muestra la triangulación de los datos de acuerdo con la percepción del profesorinvestigador y los alumnos una vez aplicada la intervención didáctica $A B P$, basada en principios de $A A$ y resultado de la aplicación y análisis del instrumento de vaciado de Aprendizaje Activo (Chirino, 2017).

Según los resultados analizados en la tabla de vaciado, el $89 \%$ de los estudiantes desarrolló aprendizaje activo por cuanto hubo una modificación en las prácticas docentes que conllevaron a los estudiantes a desarrollar habilidades para monitorear, autorregular y controlar sus aprendizajes. En cuanto al aprendizaje situado, los docente a partir de los saberes previos de los estudiantes, pudieron determinar qué sabían y qué necesitaban mejorar en la competencia específica, para proceder a explicar la metodología ABP y organizar los grupos de trabajo.

En relación con el aprendizaje social, el trabajo colaborativo propio del Aprendizaje Basado en Problemas, permitió la interacción social y académica de los estudiantes a través de la conformación de grupos y el trabajo en google drive. En este aspecto, vale la pena resaltar que respecto a la competencia transversal resolución de problemas, solo el $13 \%$ consideran que siempre (en la escala de Likert), obtuvieron el respaldo de sus compañeros en la resolución del problema, puesto que era la primera vez que trabajan de esta forma y al inicio del trabajo, hubo resistencia por parte de algunos integrantes de los equipos, por lo cual fue necesaria la mediación constante del docente y de los líderes de cada grupo.

El aprendizaje reflexivo, fue el que obtuvo el promedio más bajo, sólo el $68 \%$ de los estudiantes considera que hubo espacios suficientes de reflexión. A pesar que se abrió un foro permanente en el Aula virtual, para preguntas de reflexión y seguimiento, éste no fue suficiente. El $32 \%$ de los estudiantes considera que deben existir más encuentros presenciales para poder dialogar con sus compañeros de grupo y debatir en torno a la solución del problema, así como para acceder de forma directa a las explicaciones de la docente y poder manifestar sus dudas e inquietudes.

A continuación, la figura 3 muestra la triangulación entre la percepción del profesor respecto a los atributos aplicados en el aprendizaje activo conforme a los resultados de la lista de cotejo y la percepción de los alumnos obtenida del instrumento de Aprendizaje activo en el aula (Chirino, 2017)

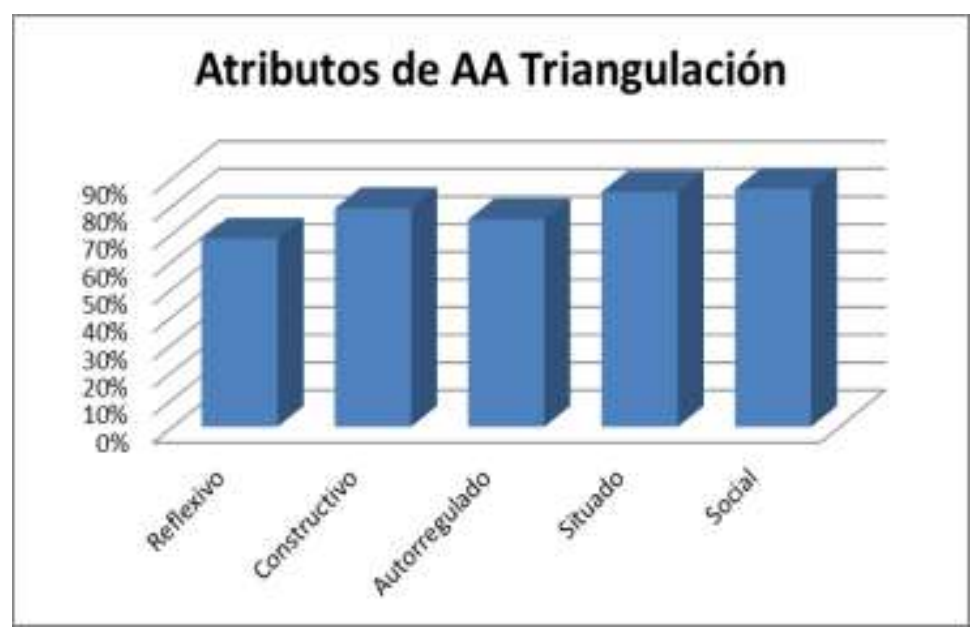

Fig. 4. Triangulación atributos AA.

Al triangular los atributos de aprendizaje activo, entre la apreciación del docente con la de los alumnos se identifica que el aprendizaje reflexivo fue el que menos se desarrolló en los estudiantes durante la intervención 
pedagógica, apenas alcanzó un $68 \%$, por lo que se hace necesario abrir más espacios para el encuentro entre profesor y alumnos, ya que dada la modalidad de Educación a distancia, éstos son cada quince días y los tiempos de clase muy cortos; el aprendizaje constructivo por su parte fue más significativo, el $79 \%$ de los alumnos integra los aprendizajes previos a su estructura cognitiva (Díaz Barriga; Hernández, G. 2004) y es capaz de emplear el conocimiento en otros contextos y en su práctica pedagógica.

En cuanto al aprendizaje autorregulado, los estudiantes en un $75 \%$ desarrollaron este atributo a través de la intervención pedagógica puesto que buscaron otras formas para comunicarse y acceder a la información y a nuevas fuentes de conocimiento (Kanfer, 1997), como alumnos de un programa de educación a distancia, han desarrollado esta habilidad a lo largo de su carrera. Los atributos de aprendizaje activo situado y social alcanzaron los más altos porcentajes, $85 \%$ y $86 \%$ respectivamente en la medida que el docente partió de la exploración de saberes previos de los estudiantes, explicó la metodología a aplicar, introdujo el tema a través de una sesión presencial y se dieron reglas claras desde un principio a través del escenario del problema; en lo social, la creación del foro, de los grupos en google drive y en whatsapp, permitió la interacción social y el trabajo colaborativo de los participantes desde un comienzo.

Los atributos de la metodología AA: constructivo, situado y social, siempre se mantuvieron en primer lugar con respecto a los demás. Los estudiantes, como pertenecientes a una modalidad de educación a distancia, mediada por las tecnologías, prefieren en su mayoría trabajar colaborativamente, aplicar los aprendizajes en situaciones específicas y aprender haciendo. $Y$ en menor porcentaje el aprendizaje reflexivo, dadas las dificultades presentadas al interior de algunos grupos y porque consideran que faltaron más espacios de socialización e interacción de forma presencial. Al ser una modalidad a distancia, los encuentros se limitan a cada quince días y para la aplicación de la propuesta, solo hubo tres encuentros presenciales, el resto de la comunicación fue por el Google Drive y a través del foro permanente del Aula Virtual.

\section{DISCUSIÓN FINAL}

El aprendizaje activo como como metodología y estrategia vinculada con el constructivismo, permitió el diseño, aplicación y evaluación de los procesos de enseñanza y aprendizaje de los estudiantes objeto de la investigación tal como lo platea Revans(1998), Además, es un aprendizaje que se visualiza a través de la experiencia y la construcción mediada por procesos de comunicación e interacción en la cual todos aprenden de manera significativa (Schwartz y Pollishuke, 1995), por medio del trabajo colaborativo dentro y fuera del aula tal como se aplicó a través de las fases del ABP, y que a su vez están directamente relacionadas con las fases de aprendizaje activo propuestas por Chirino, Ramos y Lozano (2014), con base en los estudios de Huber (2008).

La estrategia ABP permitió una modificación significativa en la práctica docente a través de la aplicación de los principios de Aprendizaje Activo, que conllevó a los estudiantes a desarrollar habilidades para monitorear, autorregular y controlar su aprendizaje. Se mejoró notablemente en la operacionalización de la didáctica de la competencia gramatical, ya que los futuros docentes crearon las condiciones idóneas para que sus estudiantes alcancen un conocimiento instrumental y cuál debe ser el tratamiento didáctico de una gramática vinculada al texto. El ABP es un método didáctico, dentro de las pedagogías activas y a su vez una estrategia de enseñanza denominada aprendizaje por descubrimiento. Para Barrows (2008), el propósito de este método es que el estudiante sea responsable de su propio aprendizaje. Por lo anterior, el rol docente debe ser facilitador de un proceso autonómico cognitivo y socioconstructivista del aprendizaje; así mismo el centro de organización y de aprendizaje reside en la proposición y solución de problemas, con el consecuente desarrollo de habilidades de pensamiento de nivel superior. Respecto a la competencia transversal resolución de problemas, con base en el nivel tres de Villa y Poblete (20017), los estudiantes mejoraron su habilidad para conseguir información y tomar decisiones colaborativamente frente a la resolución del problema, pero es preciso buscar otros espacios de reflexión para que tanto docentes como estudiantes realicen un proceso de metacognición frente a su proceso de aprendizaje.

Frente a los atributos de la metodología Aprendizaje Activo, siempre estuvieron en primer lugar lo constructivo, situado y social, por las mismas razones anteriores. Los estudiantes, como pertenecientes a una modalidad de educación a distancia, mediada por las tecnologías, prefieren en su mayoría trabajar colaborativamente y buscan nuevas fuentes de conocimiento (Kanfer, 1977), aplican los aprendizajes en situaciones específicas y aprenden haciendo; $y$ en menor porcentaje el aprendizaje reflexivo, dadas las dificultades presentadas al interior de algunos grupos y porque consideran que faltaron espacios de socialización e interacción de forma presencial. Al ser una modalidad a distancia, los encuentros se limitan a cada quince días y para la aplicación de la propuesta, solo hubo cuatro encuentros presenciales, el resto de la comunicación fue por la herramienta Google Drive y a través del foro permanente del Aula Virtual. 
En lo social, la metodología ABP parte de principios de aprendizaje colaborativo para la resolución del problema y permite según Serna y Diaz (2013) que los estudiantes tomen sus propias decisiones sin que el docente pierda su liderazgo en el aula, ya que éste debe estar al tanto de lo hacen sus estudiantes durante todo el proceso para guiarlos y reorientarlos cuando sea necesario y así lo reconoce el $100 \%$ de los estudiantes. Por su parte en el aprendizaje reflexivo según Jay y Johnson (2002), los estudiantes deberían aprender no solamente como se reflexiona sobre el objeto de aprendizaje y los propios procesos de aprenderlo, sino también sobre perspectivas o marcos alternativos y las implicaciones de lo que se aprende. Los docentes integran preguntas que orienten la reflexión al menos una vez para el plano individual y para el colectivo (Huber, 2008, p. 72). Al respecto en la reflexión los alumnos identificaron tanto hechos centrales como preguntas abiertas en relación con el objeto de aprendizaje. Lo mismo vale en cuanto a las propias ideas, emociones, resistencias, valores y preferencias. La reflexión común ayuda a que se puedan enterar de perspectivas alternativas.

A continuación se explica detalladamente los pasos para la resolución del problema específico relacionado con la competencia gramatical en $\mathrm{ABP}$, trabajo desarrollado a través del google drive

Escenario: Con base en la siguiente regla ortográfica, elaboren una Secuencia de actuación didáctica (taller) para grado $6^{\circ}$, donde propongan actividades para que sus estudiantes superen esa dificultad y que conlleven a la inferencia de la regla y a detectar sus excepciones. Así mismo una justificación a la luz de las lecturas de cómo aplicaron los principios y didáctica adecuada a esta competencia.

Regla: "Se escriben con v las palabras que comienzan por ave, avi, eva, eve, evi y evo. EXCEPTO: ebanista, abierto y abismo".

Para resolver el problema de didáctica cuentan dos semanas, cada equipo estará en libertad de ajustar su trabajo como mejor les acomode, pero asegúrense de entregar un trabajo de calidad por lo que se sugiere que sigan la siguiente metodología como se presenta en la tabla 6:

Tabla 6. Explicación del escenario del problema

\begin{tabular}{|l|l|}
\hline \multicolumn{2}{|l|}{ Metodología del Aprendizaje Basado en Problemas $(\underline{\mathrm{ABP}})$} \\
\hline Fase & Actividades \\
\hline $\begin{array}{l}\text { Definición del problema y } \\
\text { planeación de las acciones } \\
\text { (Colocar su listado en el } \\
\text { foro) }\end{array}$ & $\begin{array}{l}\text { Leer el planteamiento del problema. } \\
\text { Discutir en torno a cuál es el problema a resolver. } \\
\text { Llegar a la definición de un problema, expresado en términos de un enunciado o } \\
\text { pregunta. } \\
\text { Definir y enlistar las actividades a realizar para contar con la información suficiente y } \\
\text { necesaria para diseñar una secuencia didáctica de acuerdo a los principios de la } \\
\text { competencia gramatical en la enseñanza de dicha disciplina en el aula. } \\
\text { Distribuir dichas actividades entre los miembros del equipo estableciendo claramente } \\
\text { responsabilidades de cada quien, y tiempos de entrega. }\end{array}$ \\
\hline $\begin{array}{l}\text { Búsqueda de información } \\
\text { que necesitan para el diseño } \\
\text { todos están obligados a } \\
\text { obtener información }\end{array}$ & $\begin{array}{l}\text { Cada uno de los miembros del equipo recopila la información que le corresponde e } \\
\text { intercambia con los demás miembros del equipo dicha información. }\end{array}$ \\
\hline $\begin{array}{l}\text { Solucionar el problema } \\
\text { Se entrega en la carpeta de } \\
\text { Entregas }\end{array}$ & $\begin{array}{l}\text { Basándose en la información recopilada se diseña la secuencia didáctica de forma } \\
\text { libre. }\end{array}$ \\
\hline
\end{tabular}

\section{Organización del trabajo colaborativo:}

Cada equipo tiene su espacio de trabajo en el google docs, pero es muy importante que en el foro de equipo también se refleje el trabajo de cada semana sea éste en forma de discusión del problema, luego en enlistar las acciones e información que necesitan para resolver la tarea (dificultades gramaticales encontradas, contenidos gramaticales a abordar de acuerdo con el grado seleccionado, relación con los estándares del grado, posibles estrategias y actividades, relación con los principios y didáctica de la competencia gramatical, entre otros que consideren oportunos), traer información pertinente al equipo y compartirla con todos en el foro, para finalmente elegir la solución adecuada y plasmarla en la secuencia didáctica. 
El equipo elaborará la secuencia didáctica con la correspondiente justificación de cómo se cumplen los principios y metodología de la didáctica de la competencia gramatical a la luz de las lecturas consultadas.

El trabajo debe ser equitativo y todos los miembros del equipo deben participar en el diseño de la secuencia didáctica y la redacción de la justificación. La secuencia didáctica debe contener: Identificación, estándar, competencias que desarrolla, indicadores de logro, actividades de aprendizaje ampliamente descritas, estrategias de autodirección que fomentará, materiales didácticos (pueden ser recursos educativos abiertos o elaborados por el profesor, especificar la duración y mecanismo de evaluación del aprendizaje.

\section{CONCLUSIONES}

El objetivo de la investigación era determinar la incidencia de la intervención pedagógica Aprendizaje Basado en Problemas, sustentada en principios de Aprendizaje Activo en el desarrollo de la competencia genérica resolución de problemas y la competencia específica operacionalización de la didáctica de la competencia gramatical en estudiantes de IX semestre de una licenciatura. Con base en lo anterior se diseñaron instrumentos para evaluar cada una de las competencias mencionadas tanto antes como después de la intervención pedagógica. Así mismo, se trabajó de forma colaborativa en la resolución de un problema relacionado con la didáctica de la competencia gramatical a través de la creación de grupos en Google Drive, la apertura de un foro permanente en el Aula Virtual, además de las sesiones presenciales antes durante y después de la intervención. Por consiguiente, la importancia de la investigación radica en la necesidad de mejorar el desempeño de los futuros licenciados a través del análisis de una situación problema en la didáctica de la Lengua Castellana, para que con base en los principios del aprendizaje activo y la aplicación de los pasos de la metodología ABP, propongan alternativas de solución de manera colaborativa, mejorando su praxis pedagógica a través de metodologías alternas.

Finalmente, el trabajo colaborativo en el Google Drive resultó bastante motivante para los estudiantes, debido a que la mayoría no había utilizado esta herramienta y los que sí lo habían hecho, era para compartir información de una tarea, pero nunca para la resolución de un problema y menos como parte de la metodología del docente. Esta forma de trabajar permitió la interacción de todos y cada uno de los participantes con la asesoría del docente. A pesar de que tuvieron dificultades para iniciar las intervenciones, durante el proceso mejoraron notablemente en la operacionalización de la didáctica de la competencia gramatical, se generó aprendizaje significativo dado que hicieron uso de estrategias de aprendizaje activo e interiorizaron atributos de tipo cognitivo, procedimental y praxiológico para su desempeño profesional; a la vez que desarrollaron la capacidad de generar espacios de aprendizaje significativo permitiéndoles fortalecer las competencias comunicativas, sociales y emocionales, necesarias para su futuro laboral y profesional.

\section{REFERENCIAS}

Barrows, H. S. A. Taxonomi of Problem-Based Learnig Methods, doi.org/10.1111/j.1365-2923.1986.tb0.1386.x. Medical Education (1986)

Barrows, H. S. The Mínimum Essential for Problem-Based Learnig. Generic Problem-Based Learnig,. (2008).

Chirino, V. Aprendizaje Activo Como Estrategia Educativa. Documento de trabajo. Tecnológico de Monterrey: México (2012)

Chirino, V., Ramos A. y Lozano A. Reenfocando el Aprendizaje Activo hacia un Modelo de Aula Invertida. Pautas para la Acción Docente. Memorias del Primer Congreso Internacional de Innovación Educativa. Tecnológico de Monterrey. México (2014).

Chirino, V. Aproximaciones para la Operacionalización del Aprendizaje Activo en La Práctica. Working. Paper. Escuela de Humanidades y Educación. Tecnológico de Monterrey (2017)

Díaz Barriga, F. y Hernández, G. Estrategias Docentes para un Aprendizaje Significativo. Una Interpretación Constructivista. Ed.Trillas Editores (2004)

Escribano, A. y Del Valle, A. El Aprendizaje Basado en Problemas. Una Propuesta Metodológica en Educación Superior. Madrid, España: Narcea (2010)

Ferreira, C. y Gomes, L. Proyecto Tuning América Latina en las Universidades Brasileñas. Características y ámbito en el área de educación. Revista Paradigma, Vol. XXXIV, № 1; junio (83-96), ISSN:1011-2251,(2013)

Huber, G. Self-Regulated Learning by Individual Students. En: D.STERN \&G.L.Huber (cons), Active Learnig for Studentes and Teachers (137-158). Frankfurt:Lang (1997)

Huber, G. Aprendizaje Activo y Metodologías Educativas. Active Learning and Methods of Teaching. En Revista de Educación, número extraordinario , pp. 59-81. ISSN:0034-592X,(2008).

Jaimes, N. S. El Mejoramiento en el Desempeño de Exposiciones Orales en Inglés desde Didácticas Colaborativas del Aprendizaje Activo. (Tesis de Maestría sin publicar) Instituto Tecnológico y de Estudios Superiores de Monterrey (2014) 
Jay, J. and Johnson, K. Capturing Complexity: a Typology of Reflective Practice for Teachers Education. Teaching and Teacher Education (2002)

Kolb, D. The learning Style Inventory: Technical Manual. Boston, Ma: Mcber (1976)

Kanfer, F. Selbstmanagment- Methoden. En: F.H. Kanfer \& A.P.Goldstein (com). München:Urban \&Schawarzenberg (1977)

Koo, L. C. Learning Action Learning. En Journal of Workplace Learning, Vol 11, Number 3. ISSN:1366-5626,(1999)

McMillan, J, y Schumacher, S. Investigación Educativa. Madrid: Pearson. 5aㅡ ed. 656 p. ISBN: 978-84-205-4163-1, (2005)

Ochoa Sierra, L. La Gramática y su Relación con la Lectura y la Escritura. En: Revista Educación y Ciudad, No 15, Universidad Nacional, ISSN: 0123-0425, (2008)

Okuda Benavidez, M. y Gómez-Restrepo, C. Métodos en Investigación Cualitativa: Triangulación. Revista Colombiana de Psiquiatría. Vol. XXXIV, n 1, pp 118-124. ISSN: 0034-7450, (2005)

Peñalosa Castro, E.,- y Castañeda Figueiras, S. Generación de Conocimiento en La Educación en Línea: Un Modelo para el Fomento de Aprendizaje Activo y Autorregulado. Revista Mexicana de Investigación Educativa, 13(36), 249-281.ISSN -E2594-2271, (2008)

Revans, R.The Golden Jubilee F Action Learnig: A Collection of Papers Writting During . Manchester: Manchester Action Exchange (1998)

Riesco González, M. El Enfoque por Competencias en el EEES y sus Implicaciones en la Enseñanza y el Aprendizaje. En: Tendencias Pedagógicas $N^{\circ} 13$. DOI prefix: 10.15366/tp, (2008)

Schwartz, S. y Pollisshuke, M. Active Learnig in the Classroom:Classroom Organization. Narcea Ediciones: Madrid (1995) Serna, H. y Diaz, A. Metodologías Activas del Aprendizaje. Fundación María Cano FUMC, ISBN: 978-958-59061-1, (2013)

Rogoff, B. Observing Sociocultural Activity on three Planes: Participatory Apropriation, Guided Participaction and Apprenticeship. En J.V Wertsch, P. Del Rio y A. Álvarez (comps), Sociocultural Studies of Mind. Cambridge: University Press, (1995)

Universidad Pedagógica y Tecnológica de Colombia. Proyecto Académico Educativo (PAE). Licenciatura en Educación Básica, UPTC (2009).

Valenzuela, J. y Flores, M. Fundamentos de Investigación Educativa. Volumen 2 y 3 (Spanish Edition) (Posición en Kindle3713-3714). Edición de Kindle (2013)

Villa, A. y Poblete, M. Aprendizaje Basado en Competencias. Una Propuesta para La Evaluación de las Competencias Genéricas. Bilbao: Ediciones Mensajero, S.A.V. ISBN 978-84-271-283-0, (2007)

Villarroel, V. y Bruna, D. Reflexiones en Torno a las Competencias Genéricas en Educación Superior: Un Desafío Pendiente. En: Psicoperspectivas (vol.13 no.1), ISSN 0718-6924 electrónica,(2014)

Yániz, C. y Villardón, G. Planificar Desde Competencias para Promover el Aprendizaje. El Reto de la Sociedad del Conocimiento Para el Profesorado Universitario. Cuadernos Monográficos del ICE, N 12.Bilbao: DEUSTO digital, (2006) 